\title{
Rêves de fleurs et rêves de fruits : une construction andine du genre
}

Palmira La Riva González

\section{OpenEdition}

Journals

Édition électronique

URL : https://journals.openedition.org/jsa/11557

DOI : 10.4000/jsa. 11557

ISSN : 1957-7842

Éditeur

Société des américanistes

Édition imprimée

Date de publication : 5 décembre 2010

Pagination : 181-203

ISSN : 0037-9174

\section{Référence électronique}

Palmira La Riva González, "Rêves de fleurs et rêves de fruits : une construction andine du genre », Journal de la Société des américanistes [En ligne], 96-2 | 2010, mis en ligne le 10 décembre 2014, consulté le 02 septembre 2022. URL : http://journals.openedition.org/jsa/11557 ; DOI : https:// doi.org/10.4000/jsa.11557 


\title{
RÊVES DE FLEURS ET RÊVES DE FRUITS : UNE CONSTRUCTION ANDINE DU GENRE
}

\author{
Palmira LA RIVA GONZÁLEZ*
}

De nombreux travaux sur le genre ont montré que les comportements et les rôles attribués aux hommes et aux femmes sont des constructions culturelles variant dans le temps et dans l'espace. Les représentations du genre sont par ailleurs étroitement liées aux conceptions du corps et de la personne. Notre intention est de montrer comment, dans le cas de la représentation du genre que se font les populations des Andes du Sud, l'opposition masculin/féminin, tout en étant fondée sur un aspect biologico-physiologique, exige d'être renforcée au cours des premières étapes de vie de l'individu, à la fois par le discours et par des pratiques rituelles qui modifient la nature même du corps. [Mots-clés: Andes, Pérou, genre, représentation du corps, pratiques rituelles.]

Soñando con flores y frutas. La construcción andina del género. Una abundante literatura sobre género ha puesto en evidencia que los comportamientos y roles atribuidos a hombres y mujeres son construcciones sociales que varían con el tiempo y la cultura. Estas representaciones de género están por lo demás estrechamente asociadas a las concepciones del cuerpo y de la persona. Nuestra intención es mostrar como, para las poblaciones de los Andes del sur peruano, la oposición hombre/mujer a pesar de tener como base una explicación "físico-biológica» debe, sin embargo, ser reforzada durante la primera etapa de la vida de los individuos a través del discurso y de prácticas rituales que modifican la naturaleza misma del cuerpo. [Palabras claves : Andes, Perú, género, representación del cuerpo, prácticas rituales.]

Dreams of flowers and fruits. The Andean construction of gender. A rich literature about gender has demonstrated that the roles and behaviours assigned to men and women are social constructions varying within and across cultures and changing over time. These representations of gender are tightly related to the conceptions of the body and personhood. Our intention is to demonstrate how, in the Southern Peruvian Andes, the man/woman opposition, although having a biological explanation, has to be

* Doctorante à l'université Paris Ouest Nanterre La Défense, Laboratoire d'ethnologie et de sociologie comparative, Maison René-Ginouvès (archéologie et ethnologie), 21 allée de l'université, 92023 Nanterre [palmirakis@hotmail.com].

Journal de la Société des Américanistes, 2010, 96-2, pp. 181-203. O Société des Américanistes. 
reinforced during the beginning of the life cycle of every person, by discourse and ritual practices modifying the body. [Key words : Andes, Peru, gender, representations of the body, ritual practices.]

\section{INTRODUCTION}

Les recherches effectuées au cours des quarante dernières années sur la représentation des différences entre les hommes et les femmes ont démontré le caractère éminemment culturel des représentations de la personne sexuée ${ }^{1}$. Elles ont également montré en quoi les comportements et les rôles attribués à chacun des deux sexes sont des constructions sociales qui traduisent des rapports de subordination, de hiérarchie ou d'inégalité, révélant des enjeux de pouvoir. Margaret Mead (1963 [1928]) fut la première à introduire l'idée d'une composante culturelle, et pas seulement biologique, dans la façon d'appréhender la différence sexuelle. Michel Foucault, dans son Histoire de la sexualité (1984), a mis en évidence l'étroite relation entre le contexte historique et les représentations du corps et de la sexualité. Récemment, certains auteurs sont allés jusqu’à dénoncer le caractère illusoire des différences biologiques et physiologiques entre les hommes et les femmes, ces différences n'étant que des constructions sociales et culturelles, variant dans le temps et dans l'espace géographique, mais légitimant des rapports de domination ${ }^{2}$. Pour Bourdieu (1998, pp. 13-21), « la définition sociale du corps masculin et féminin n'est pas du tout un simple enregistrement des propriétés anatomiques et naturelles, c'est une construction fondée sur l'accentuation de certaines différences et le voilement de certaines similitudes ». L'approche anthropologique d'Héritier (1996, pp. 32-39), tout en plaçant les données biologiques à l'origine de la construction différenciée des sexes, confirme leur caractère de "construction culturelle ${ }^{3}$. De leur côté, Godelier et Panoff (1998) ont analysé les correspondances entre les représentations du corps, les rapports entre les sexes et les formes de pouvoirs et de hiérarchies propres à une société en Océanie. Les études andines relatives au genre - envisagé dans un sens très large - ont été marquées par une certaine idéalisation de la complémentarité et de la dualité andines, ce qui a eu pour conséquence de masquer les enjeux de pouvoir sous-jacents aux rapports sociaux entre les sexes. Cependant, ces dernières années, nous assistons à une reprise critique du dualisme andin, qui a permis de faire ressortir les rapports inégaux entre hommes et femmes ${ }^{4}$. À côté de théories sur le parallélisme ou la complémentarité entre hommes et femmes, des théories concernant la subordination ou la complémentarité hiérarchique ont connu un certain développement ${ }^{5}$.

Notre étude ne porte cependant pas sur les implications sociopolitiques de la construction du genre, ni sur les rapports entre les représentations du corps et l'ordre social. Ce qui nous occupe ici, et qui nous paraît fondamental pour comprendre les rapports entre hommes et femmes, qu'ils soient égaux ou inégaux, 
ce sont les relations entre, d'une part, les représentations du corps et de la personne et, d'autre part, la construction sociale du genre au cours des premières étapes de vie de l'individu ${ }^{6}$. En effet, cet aspect de la construction du genre n'a pas été traité ou l'a très peu été. Notre analyse portera sur les pratiques rituelles et les discours relatifs au corps et à son fonctionnement, ainsi que sur les différences physiologiques et psychologiques entre les hommes et les femmes, telles qu'elles sont exprimées par nos interlocuteurs. Nous nous intéresserons essentiellement aux représentations et aux pratiques relatives à la grossesse, à la coupure du cordon ombilical, au traitement du placenta et aux discours sur la différence essentielle entre hommes et femmes.

Les données ethnographiques ont été recueillies dans la communauté de Surimana ${ }^{7}$ (province de Canas, département de Cuzco) où nous travaillons depuis 1998. Nos interlocuteurs sont des femmes, des enfants et des hommes de la communauté, parmi lesquels se trouvent des spécialistes rituels et des accoucheurs traditionnels, aussi bien que des agents de santé communautaires ${ }^{8}$. Surimana dispose d'une modeste infirmerie ${ }^{9}$, d'un technicien de santé et de deux infirmières. La province de Canas, qui fait partie du réseau de santé Canas Canchis Espinar (trois provinces limitrophes) du ministère de la Santé ${ }^{10}$, ne compte qu'un seul centre de santé dans la capitale provinciale et onze infirmeries rurales. Le service officiel de médecine est précaire, car on ne compte qu'un médecin pour 6000 habitants ${ }^{11}$.

Les habitants de Surimana sont de langue quechua, mais beaucoup d'entre eux, surtout les jeunes et les hommes, s'expriment couramment en espagnol, étant donné leurs contacts étroits lors des transactions commerciales ou administratives avec les villes de Combapata et de Cuzco. Ils pratiquent essentiellement une agriculture de subsistance, une faible partie de la production étant destinée à l'échange et au marché local.

\section{LA REPRÉSENTATION DU GENRE DANS LE MONDE ANDIN}

$\mathrm{Si}$, dans les populations andines quechuaphones, le terme ruma ${ }^{12}$ définit l'être humain de façon générale et s'applique tant aux hommes qu'aux femmes, des termes plus spécifiques sont utilisés pour parler des sexes, qhari pour les hommes, warmi pour les femmes ${ }^{13}$. Le genre est ici envisagé à partir de différences biologico-physiologiques, sans toutefois accorder le primat au biologique. Ces différences déterminent la constitution animique et psychologique des hommes et des femmes; de fait, il existe une étroite relation d'interdépendance et de continuité entre les composantes matérielles et spirituelles du corps.

Dans les études sur le cycle de vie dans le monde andin, un rôle central est attribué au rituel de la coupe des cheveux (rutuchikuy), considéré comme déterminant la différenciation entre les sexes (Christinat 1989 ; Harris 1980). En effet, 
après la coupe des cheveux qui a lieu à un âge compris entre deux et quatre ans, une différenciation par le vêtement est clairement imposée au petit garçon et à la petite fille. Mais les matériaux que nous avons recueillis sur notre terrain révèlent une réalité plus complexe. Nous avons pu observer que la distinction entre les sexes, loin de se réduire à un moment privilégié, correspond à un long processus de construction de l'identité sexuelle qui commence avant même la naissance de l'individu, lors des rêves prémonitoires annonçant la gestation d'un enfant. Ce processus se poursuit durant la grossesse, passe par la coupure du cordon ombilical, par le traitement donné au placenta et culmine en effet avec la coupe des cheveux, pour se prolonger à travers la socialisation des hommes et des femmes tout au long de leur vie ${ }^{14}$.

\section{Rêver}

" Le monde des rêves nous offire l'occasion, si fugitive soit-elle, de converser avec nos amis lointains, nos morts, et nos dieux » (Dodds 1966, p. 102). L'expérience onirique joue un rôle fondamental dans la vie des populations sud-andines péruviennes, conditionnant et déterminant les actions et décisions de la vie quotidienne. Elle est aussi une partie essentielle des processus d'initiation des spécialistes rituels et constitue un moment privilégié de rencontre avec les êtres de l'autre monde. On peut repérer deux catégories de rêves : ceux dits " prémonitoires » qui annoncent un événement futur et ceux dits " oraculaires » qui permettent d'entrer en communication avec les dieux, les morts ${ }^{15}$, les esprits ou des puissances surnaturelles ${ }^{16}$.

Parmi les rêves prémonitoires, les interlocuteurs font une distinction entre les bons et les mauvais rêves. Les bons ont une origine divine, ils proviennent de Dieu, et les mauvais ont une origine diabolique. Dans la catégorie des mauvais rêves, nous avons les rêves qullu "frustration, extinction ». Dans le contexte de l'expérience onirique, ce terme peut être traduit par "de mauvais augure", sans que le rêve ait pour autant une origine diabolique. Le terme qullu- ${ }^{17}$, en tant que verbe, fait référence à l'extinction des humains ou des ressources et au non-accomplissement ou à la frustration des objectifs ${ }^{18}$.

Ishkay claseyá sueñu : Diosninchispa partinmanta sumaq sueñu, sumaq t'ikata rikunki, imatapas allin sueñuchata sueñukunki chayqa... mañakumki diosmanta chayqa, allinpaq sumaq sueñu hamushan. Sichus mana allin sueñukunata creenki, satanaspa partinmanta kallanpunin chay sueñukunki, cayesunki. Imapas pasasunki. [...] Chay musquyqa ciertun. Diosmanta hamun riki. Allin musquy hamun Diosmanta kaspa, millaymusquytaq hamun diabluq partinmanta, satanasmanta. [ Il y a deux sortes de rêves : ceux qui proviennent de notre Dieu sont de beaux rêves, tu vois des fleurs. Si tu fais un bon rêve... si tu as demandé quelque chose à Dieu, il te vient un rêve de bon augure. Si tu crois aux mauvais rêves - il y en a qui viennent de Satan - si tu en fais, ça t'arrive. Quelque chose t'arrive. Les rêves sont vrais. Ils proviennent de Dieu. Les bons rêves viennent parce 
qu'ils viennent de Dieu, et les mauvais rêves viennent du diable, de Satan. »] (Saturnino Mamani, spécialiste rituel ou paqu et accoucheur)

Les rêves prémonitoires peuvent annoncer des événements positifs ou négatifs, intéressant non seulement le rêveur mais aussi la communauté tout entière.

Chaque individu possède sa propre clef des songes, avec un répertoire spécifique de rêves et de significations/interprétations qui peut varier tout au long de sa vie. Ce caractère individuel des significations attribuées aux symboles oniriques est souvent souligné par les informateurs :

Manan musquyta igualchu sapanka runaman, sapanka ruman creen imayna musqumantapis. Chaynata musqukumku, entonces sapanka runan sapanka warmipas diferente musquykuna [...]. [ [ Les rêves ne sont pas les mêmes pour chaque personne, chacun croit selon ses rêves. C'est ainsi qu'ils rêvent, pour chaque personne : pour chaque femme, les rêves sont différents (...). »] (Francisco Quispe, agent de santé communautaire)

Toutefois, malgré ce caractère individuel, l'expérience onirique relève aussi d'un système culturel et historique (Dodds 1966) ${ }^{19}$. En effet, pour certains rêves prémonitoires, il y a un fond commun de symboles et d'images codifiés dont l'interprétation est partagée par tous les membres de la communauté. C'est notamment le cas des rêves annonçant la naissance d'un enfant : rêver de fleurs renvoie à la fertilité et à la reproduction (miray), aussi bien des animaux que des êtres humains ${ }^{20}$. Les fleurs sont toujours présentes lors de certains rites de passage, comme le umuchay " l'eau de secours ». Il s'agit d'une pratique rituelle domestique qui s'effectue peu de jours après la naissance de l'enfant et qui consiste à lui appliquer de l'eau d'une source à laquelle on ajoute du sel afin qu'il se transforme en runa, c'est-à-dire en être humain. Il y a aussi des fleurs lors de la coupe des cheveux, au cours de mariages, de rites propitiatoires de fertilité des animaux $^{21}$ et dans le rituel thérapeutique d'appel à la force vitale, animu waqyachiy. Relevons que, dans le récit de Saturnino Mamani, spécialiste rituel et accoucheur, le dormeur « voit des fleurs dans les rêves, provenant de Dieu » ${ }^{22}$.

T'ikachata rikumku musqurumku chayqa, ninku t'ikatan, t'ikata rikunku wakin casupin t'ikata rikunku uywapaq, animalpaq, wakin casupitaq t'ikata rikunku, wawayuq kanankupaq, " seguramente unquq kasaq, chaypaqmi t'ikata musquruni » nispa. ["Si on voit, on rêve de fleurs, dit-on, si on voit des fleurs, dans certains cas on voit des fleurs (et cela annonce la reproduction) des animaux, dans d'autres cas on voit des fleurs et ça annonce qu'on va avoir des enfants : "Peut-être vais-je tomber enceinte, c'est pour cette raison que j'ai rêvé de fleurs”, dit-on. »] (Francisco Quispe)

Les rêves peuvent donner des informations sur le sexe de l'enfant à naître. Par exemple, si rêver de fruits renvoie certes à la fertilité, rêver de fruits avec un noyau ou des pépins annonce la naissance d'un garçon ${ }^{23}$, alors que rêver de fleurs annonce la naissance d'une fille ou le fait d'avoir un filleul ${ }^{24}$. 
Ima frutachata rikunku qhari wawa kananpaq. T'ikachakunata rikunku, ima colorchata, warmi wawayuq kananpaq. ["Si l'on voit en rêve un fruit, cela annonce un garçon. Si l'on voit des fleurs de n'importe quelle couleur, c'est pour avoir une fille. »]

Flores, las t'ika, significa que vas a tener un ahijado o va a ser mujer: [" Rêver de fleurs signifie que tu vas avoir un filleul ou une fille. »]

Soñar con frutas viene a ser que vas a tener un hijo varón o vas esperar de acá a un tiempo un hijo. La palta, el mango : hombre. Cuando tienen pepa, su qoroto, es varón ${ }^{25}$. Chayta ninku nispa : "Qhari wawayki kanqa, platano también [...] [« Rêver de fruits veut dire que tu vas avoir un garçon bientôt. L'avocat et la mangue annoncent la naissance d'un garçon. Quand les fruits ont un noyau, son $q^{\prime}$ uruta $^{26}$, c'est un garçon. On dit cela. Pour avoir un garçon : des bananes aussi (...). »] (Juana Turpa)

Les fruits à noyau et à pépins sont étroitement associés au sexe masculin en raison de la valeur génésique attribuée aux graines $m u h u^{27}$, terme qui fait aussi référence au sperme ${ }^{28}$. Il en est de même en aymara, le terme jatha désigne à la fois le sperme et les graines (Arnold et al. 1999, p. 107).

La conception d'un enfant est considérée comme la rencontre de la semence de l'homme (muhu) avec le sang menstruel (killa yawar) de la femme. Les personnes avec lesquelles j'ai travaillé disent en effet que la semence de l'homme « se cuit » et/ou " croît » dans le sang menstruel, la substance masculine étant perçue ici comme un aliment. La gestation peut être aussi perçue comme la coagulation du sang féminin par le sperme (La Riva 2000, p. 171). Mes interlocuteurs pensent aussi que la semence est comme un petit ver (kuruchahina) qui frappe et qui est pris (hap'irapun) par l'ovaire de la femme. Dans ce cas, on peut considérer qu'il s'agit d'un écho aux discours médicaux tenus par les agents de santé communautaires et par le personnel des centres officiels de santé publique. Ceux-ci introduisent des notions de médicine allogène que les populations andines se réapproprient en les adaptant à leurs propres représentations ${ }^{29}$. C'est ainsi que, parmi les termes employés pour se référer à la semence masculine, on trouve espermatozoide emprunté à l'espagnol :

Espermatozoide ninchis chayqa lichihina haykushan, anchay chayqa chay chokan, chayqa chay lichihina hamushan chaypis kuruchakuna kashan [...]. [" Ce qu'on appelle spermatozoïde, ça rentre comme du lait, ainsi cela cogne, frappe. Cela arrive comme du lait, on dit que, dedans, il y a des petits vers (...). »] (Alejandrina Caylla)

Le fœtus se forme progressivement à partir du sang. Les os n'apparaissent qu'entre le troisième et le cinquième mois, surtout dans le cas des garçons. C'est pour cette raison que, lorsqu'une fausse couche se produit dans les trois premiers mois, on ne perçoit que du sang :

Alejandrina Caylla [AC]: Wiksanchispi. Manachu hina tullukumaqa kanmanchu. Manachu hina, yawarllan. Abortanki chaypas, iskay killayoqta, huk killayuqta yawarllatan wikch'ummehis. Manan wawaqa tulluyuqraqchu. [" Dans notre ventre je crois qu'il ne peut y avoir des os. Je ne le crois pas, seulement du sang. Lorsqu'on 
fait une fausse couche à un mois ou deux, on n'expulse que du sang. Le bébé n'a pas encore d'os. ")]

Palmira La Riva [PLR] : Hayk'aqmanta tullu formakum ? [ À partir de quand se forment les os?"]

[AC] : Tulluqa formakum riki desde tawa, pisqa killayoqmanta, o sea kinsa killayoqmanta wichayman. [" Les os se forment à partir de quatre, cinq mois. C'est-à-dire à partir de trois mois. »] fœtus.

C'est la rencontre du sperme et de l'ovaire qui déclenche la constitution du

Taytaqmantaqa chay espermatozoide, leche hina haykusham, anchay chay chocam. Chay leche hina hamushan anchaypis kuruchakuna hina kan. Warmiqtaq kashan chay' ovarionchis uhupi chayqa aknata funcionashan, entonces chay kuruchakuna allin kallpayoq chayqa, haykurkun chayqa ovario hap'irapun aknata, chayqa chay warmiqwan chokarapun chaypitaq wawa formakun, chayqa kinsa unchaymantaqa yawarwañ̃a haykupum. Chayqa yawarwan runaña formakum. ["Le sperme vient du père, il rentre comme du lait et cogne. C'est comme du lait et l'on dit que la dedans il y a comme de petits vers. Et la femme a son ovaire, c'est ainsi que cela marche. Quand les petits vers ont beaucoup de force, ils rentrent rapidement et l'ovaire les capte. C'est lors de cette rencontre, se cognant avec l'ovaire de la femme, que se forme le bébé, après trois jours arrive le sang. C'est avec le sang que se forme la personne. »] (Alejandrina Caylla)

La présence du sang semble marquer le statut de personne du fœtus. Nous dirions plutôt son statut de " proto-personne " car, comme nous l'avons mentionné plus haut, il va falloir effectuer un rite de passage nommé umuchay (l'ondoiement) à la naissance pour le transformer en une personne à part entière ${ }^{30}$. À Surimana, le fœtus qui mûrit normalement dans le ventre de la mère n'est pas considéré comme dangereux et agressif pour la mère, comme ill'est dans l'ayllu macha au Nord Potosí étudié par Platt (2001). En revanche chez les Macha, comme à Surimana, tout nouveau-né est considéré comme étant saqra, c'est-à-dire diabolique et sauvage, jusqu'à ce qu'on lui attribue un nom lors du rite d'ondoiement immédiatement après la naissance.

Garçons et filles sont donc annoncés par des symboles oniriques variant selon le genre. Symboles de fertilité, les fruits et les fleurs préfigurent la naissance des êtres humains, établissant ainsi une continuité d'essence entre la nature végétale et celle des hommes.

\section{Comportement différencié du fxutus dans le ventre de la mère}

La différence sexuelle et de genre se définit avec beaucoup plus de précision dans le ventre de la mère, car les fœtus masculin et féminin diffèrent autant par leur constitution physiologique que par leur comportement qui n'est que la conséquence directe de cette constitution. Toujours selon mes interlocuteurs, les garçons prennent forme plus promptement que les filles. Ils se solidifient et 
commencent à jouer au bout de deux ou trois mois. Les filles, en revanche, mettent autour de cinq ou six mois, parfois même huit mois, pour se former. Au bout de trois mois, elles ne sont encore que du sang ou yawarllaraq ${ }^{31}$.

Contrairement aux garçons, les filles ne jouent pas beaucoup car elles n'ont pas assez de force ( $k a l l p a$ ). Ce dernier terme fait référence à la vitalité, à l'énergie qu'exigent notamment les activités physiques. Dans le cas d'un avortement, s'agissant d'une fille, Alejandrina Caylla explique qu'« on ne le sent même pas... ». La plupart des personnes rencontrées insistent sur la différence entre le foetus d'un garçon et celui d'une fille.

Qhari casupi kinsa killaqta ña riparakunña qharicha kasqa. Warmicha kaqtinmi ichaqa mana riparakuwaqchu porque yawar k'umpa hinallaraq, yawar k'umpalla. Yaqa iskay' killapi aknalla mana riparakunchu qharipis warmipis. Qharicha ichaqa kinsa chunka p'unchawpiqa casi ña riparakunña qharicha kasqa. Manan kikillan kashan. Diferentechapuni. Entonces maypichachus huk señora abortota qun iskay killayuq o kinsa killayuq chayqa, llaqapis yawar k'umpallata wikch'un. Maypichachus qharicha kaqtin ichaqa furmachayuq lluqsipun, abortan, chaypi tariyku warmi kasqa, qhari kasqa, riqsikun chay abortaq tiempun [...]. [" Dans le cas de garçons, au bout de trois mois, on voit que c'est un garçon. Mais, si c'est une fille, on ne le distingue pas car la fille n'est qu'un caillot de sang. Au bout de presque deux mois, cela ne se voit pas si c'est un garçon ou une fille. Cependant s'il s'agit d'un garçon, au bout de 30 jours on voit que c'est un garçon. Ce n'est pas pareil du tout. Quand la femme fait une fausse couche à deux ou trois mois, elle rejette juste un caillot de sang. Mais, si c'est un garçon, il est déjà formé, elle avorte et, là, on voit que c'était une fille ou un garçon. On le reconnaît au moment de la fausse couche (...) »] (Alejandrina Caylla)

Si le fotus d'une fille n'est qu'un caillot de sang, celui d'un garçon, en revanche, présente une forme précise et se comporte de manière décidée. On dit qu'il est comme un huq'ullu, un «têtard », agitant sa queue ${ }^{32}$. Il a plus de force, il bouge, il se secoue, il joue :

Chay iskay killa, kinsa killapis warmi wawaqa wiksapiqa yawarlaraq yawar k'umpalla. Yawar k'umpalla warmiqa kaq kasqa. Warmi wawaqa manan pukllaqchu kasqa [...] manan imapis riparakunchu, manan imapis riparakunchu, akna yawar k'umpalla. Aburtasqa rikuni manan kikillan... manan kikillanchu, manan agualchu fetuqa, manan igualchu. [...] Qharicha icha yasta formasqaña huq'ullu-hina, huq'ullucha-hina chupachayuq igualchalla.[...] Qhari wawa qharikunaqa aswan kallpayuq, fuerte qharikunaqa. [" Au bout de deux ou trois mois, la petite fille dans le ventre n'est que du sang, juste un caillot de sang. L'enfant n'est qu'un caillot de sang. La petite fille ne joue pas. (...) On ne voit rien du tout. Juste un caillot de sang. J'ai vu une fausse couche, ce n'est pas pareil, le foxtus [du garçon et de la fille] n'est pas pareil, il n'est pas pareil. Le garçon, lui, est déjà formé, comme un têtard, avec une petite queue, pareil. (...) Les garçons ont plus de force, ils sont plus forts les garçons. »] (Alejandrina Caylla)

Nous voyons donc que la différence sexuelle et de genre se manifeste tout au long du processus de gestation, dès les trente premiers jours de la conception. 


\section{La coupure du cordon ombilical}

Bien que les différences de constitution et de comportement entre un fœtus masculin et un fœtus féminin peuvent être annoncées, grâce à des rêves prémonitoires, avant même la conception, puis pendant la grossesse, elles doivent être renforcées et accentuées par des pratiques rituelles. Ainsi, la coupure du cordon ombilical est un geste rituel fondamental, car il détermine aussi bien les potentialités et les capacités reproductives que le comportement sexuel du futur adulte. Nous ne retiendrons ici que les aspects concernant la différenciation sexuelle que la coupure du cordon renforce. L'endroit de la coupure varie en effet en fonction du sexe de l'enfant. Ce geste est exécuté avec soin par l'accoucheur ou la sage femme qui tient compte des instructions des parents :

Palmira La Riva [PLR] : Quand on coupe le cordon ombilical, quelle est la mesure adéquate?

Ana Huallpa, accoucheuse $[\mathrm{AH}]$ : Quatre doigts ou trois doigts.

[PLR] : Et quand on coupe plus long?

$[\mathrm{AH}]$ : Pour un garçon ce serait trop long.

[PLR] : Qu'est-ce qui serait trop long?

$[\mathrm{AH}]$ : (rires) On dit que son sexe serait trop grand, trop long. Parfois si tu coupes tout petit, on croit, on dit que, si c'est une femme, son vagin va être petit et elle ne pourra pas accoucher. D'autres parents suggèrent de « le couper grand ». Pour eux, je coupe quatre doigts tant pour les hommes que pour les femmes. Évidemment, en fonction de la coupe, ils vont pouvoir engendrer après. (Entretien traduit directement de l'espagnol)

En ce qui concerne le garçon, la coupure du cordon détermine la taille du sexe. Il semble y avoir une continuité d'essence entre le cordon ombilical et le sexe masculin, car le terme servant à désigner le premier, ch'unchulin, peut aussi être employé pour faire référence au sexe masculin ${ }^{33}$. Chez la femme, une coupure trop courte pourrait avoir des conséquences sur la taille de son utérus le rendant beaucoup trop petit et, donc, empêchant la procréation. Cet acte rituel ne détermine pas seulement la capacité procréative de l'individu, mais aussi son comportement sexuel, car, selon mes interlocuteurs, si l'on coupe trop long, l'homme sera « un coureur de jupons » et la femme une « libertine ».

\section{Un traitement différencié donné au placenta}

Le placenta, paris (pares en espagnol) - terme signifiant « membre d'une paire, double, pendant » (Itier communication personnelle) -, est une des entités corporelles les plus complexes du système de représentation du corps dans les Andes : il est considéré à la fois comme le double de l'enfant, mais aussi comme 
celui de la mère, d'où son ambigüité et son caractère dualiste. Il y a aussi un rapport étroit entre le placenta, d'une part, et la peau de la mère et du nouveau-né, d'autre part ${ }^{34}$. Une fois sorti du ventre de la mère, le placenta, bien lavé, doit être brûlé ou enterré. Sans quoi, toujours vivant, il pourrait vouloir réintégrer le corps de la femme. Mes interlocuteurs disent alors que parisninchis kawsanmi [« notre double vit »]. Il est donc nécessaire de l'enterrer pour qu'il meure. Le placenta est assimilé à l'âme d'un mort, mais toujours vivant. Il pourrait se réintroduire dans le ventre de la mère, lui causant le « mauvais air " ${ }^{35}$ qui produit un gonflement du corps ou provoque la mort ${ }^{36}$.

Lavé ou brûlé, puis enterré avec beaucoup de précautions, le placenta est traité différemment selon qu'il s'agit d'un garçon ou d'une fille. En effet, le placenta, considéré comme l'alter ego de l'enfant, préfigure la vie de celui-ci. S'agissant d'une fille, le placenta est enterré avec des métiers à tisser, avec de petites casseroles, avec tout ce dont elle aura besoin pour sa vie de femme. Dans le cas d'un garçon, on enterre le placenta avec les outils du travail agricole, le chakitaqlla (bêche à pied), des instruments de musique...

Préfiguration de la vie de l'enfant, l'enterrement du placenta peut être vu aussi comme l'enterrement d'un corps qui doit mourir ou se séparer définitivement de son double après la naissance. Le placenta reçoit un traitement qui ne manque pas de rappeler, dans certaines de ses phases, celui donné au cadavre : lavage, crémation, enterrement. Dans le cas du placenta, ces précautions servent surtout à protéger la mère de ses attaques et de son désir de réintégrer son corps ${ }^{37}$.

De plus, des manipulations rituelles particulières sur le placenta peuvent permettre d'obtenir un futur enfant du sexe opposé. Par exemple, si on a eu un garçon et que l'on veut que le prochain soit une fille (ou vice versa), le placenta doit être retourné et brûlé ou enterré avec des fleurs, représentant la fertilité en général. Le retournement du placenta est considéré comme un cambio de suerte ("changement de sort ou de destinée ») qui aura un effet sur le sexe d'un futur enfant.

Ah, cuando quieres una mujercita o un varoncito, se voltea la placenta con suflorcita. Y eso se quema derechito nomás. ["Quand tu veux une petite fille ou un garçon, on retourne le placenta avec des fleurs et tu le brûles aussitôt. »] (Ana Huallpa)

Principe génésique, le placenta continue à opérer au-delà de la naissance de l'enfant. Il est un lien, un passage vers le futur.

\section{ANIMU ET CONSTRUCTION SOCIALE DU GENRE}

Dans les pratiques et discours quotidiens concernant le corps et la personne, l'être humain est perçu comme ayant un corps et un principe vital qui l'anime, animu (ánimo en espagnol), âme ou esprit, ces termes étant interchangeables ${ }^{38}$. 
L'ombre, llanthu, comme entité-reflet du principe vital est aussi mentionnée. Lorsqu'on discute des spécificités des hommes et des femmes quant à leur constitution physico-psychique, leur tempérament et leur caractère, nos interlocuteurs, quel que soit leur sexe, sont d'accord pour dire que la quantité de substance animante dans le corps ou "âme force vitale», pour reprendre l'expression de Robin (2008), varie selon le sexe et que cela explique les différences d'ordre physiologique et psychique. Selon ces personnes, les femmes possèdent une plus grande quantité d'animu que les hommes. On donne le chiffre de sept pour les femmes et de trois ou d'un seul pour les hommes ${ }^{39}$.

On ne spécifie pas les termes pour les sept animu des femmes, même si ce sont bien ces animu qui déterminent leur personnalité et expliquent leurs tendances à être plus susceptibles, plus sensibles, plus irascibles et vulnérables que les hommes :

Hay veces débil kanchis. Warmiqa imamantapis facilllata qharikuna engañaruwanchis. En cambio, enamorakuypi imata prometeruwanchis riki, qharikuna. Chayqa warmiqa " cierto " nispa creenchis riki. Hinaspa yasta huk qhariqa aprovechawanchis, chaypi chayqa anchaypi chay a veces pasan. Kunan aknata wawakumatapis wikch'upumku wacharachispa. Chaymantaqa huk taytaqa hamurullantaq: " ya nuqa prometesayki wawaykita reconocesaq, uywasaq". Imaymanata, uf caramba, ay si! " cielotapis wicharusaq. Nuqa imata qusayki que esto aquello", prometesumki. Cierto warmiqa creellanchistaq. Chayqa hukwanpas yapayukullantaq. Anchayyá chay warmi debil kanchis. Chayrayku qanchis animuyuq kanchis. Y también warmi facilta imamanpis comprometekunchis, reneganchis millayta riki, millayta reacionanchis. Chayqa imamanpis maqanakuyman hucha tariymanpas facilta haykurunchis. Anchaywan chay qanchis animuyuq ninku. ["Quelquefois, nous (les femmes) sommes fragiles. Les hommes nous trompent facilement. En amour, ils te promettent des choses, n'est-ce pas ? Alors nous leur faisons confiance. Et ils profitent de nous, ces choses-là arrivent parfois. Ils te mettent enceinte et ils t'abandonnent avec tes enfants. Après ça, arrive un autre homme : "je suis là, je te promets que je vais reconnaître ton fils, je vais m'occuper de lui, bla, bla (...) Beaucoup de choses (...) oui, hélas ! Jusqu'au ciel je peux aller pour toi" disent-ils. "Je te donnerai n'importe quoi, ceci et cela (...)". Et, nous, les femmes, nous les croyons. Et, nous avons un enfant de plus ! Nous sommes faibles. C'est pour cette raison que nous avons sept animu. Nous assumons des responsabilités, nous nous fàchons, nous avons des réactions violentes. Ainsi nous nous mettons dans des problèmes, nous nous battons. C'est pour toutes ces raisons qu'on dit que nous avons sept amimu. »] (Alejandrina Caylla)

Du point de vue physiologique, la présence de l'animu chez la femme est dû au sang qui, chez elle, est abondant et jusqu'à un certain point renouvelable, ainsi que le prouve la régularité des menstruations, même si, d'après les personnes interrogées, il est admis que la quantité de sang dans tout corps est limitée. Toutefois, ces mêmes personnes disent que certaines substances alimentaires aident à reproduire (mirachi-) ${ }^{40}$ le sang dans le corps. Parmi ces substances, nous trouvons la bière de maïs et d'autres utilisées dans la médecine officielle, comme le sérum physiologique. 
L'abondance d'animu et de sang chez les femmes fait qu'elles sont considérées comme étant q'uñi ("chaudes») par rapport aux hommes qui sont chiri (« froids $"){ }^{41}$ selon le système de classification binaire fondamental de la pensée andine, qui oppose le chaud au froid, le féminin au masculin.

On notera que les caractéristiques tant physiologiques que psychologiques attribuées aux hommes et aux femmes en raison de la présence d'animu dans les corps ne sont pas dénuées de contradictions, ce qui pose un problème quand on cherche à donner une définition univoque du concept d'animu en tant que substance ou principe vital.

Nous avons vu que la différence des genres se manifeste avant même la conception de l'enfant et se poursuit pendant la gestation du fœtus. Or, si les femmes ont une plus grande « quantité » de substance animante que les hommes, on serait tenté de penser que les femmes se forment plus rapidement dans le ventre de la mère et $\mathrm{y}$ " jouent » davantage, pour employer l'expression andine habituelle. Pourtant on a vu que ce sont là des caractéristiques attribuées, du moins à Surimana, aux garçons.

Il semblerait, de plus, que l'animu, en tant que substance corporelle étroitement associée à la présence et à la circulation du sang dans le corps, doive activer non seulement la force physique et spirituelle et les fonctions physiologiques, mais aussi avoir une certaine action sur la sensibilité et la personnalité de l'individu. Or les témoignages relevés ne sont pas toujours concordants. À première vue, ils varient en fonction du sexe des témoins. D'après un paysan, les femmes seraient plus «fortes, déséquilibrées et fofolles », alors que les hommes seraient plus " humbles».

Chay qanchis animuyuq huk siñura kanman, qharikunataqsi kanman pisqalla. Warmikunaq qanchis, chaysi siñorakuna kankumás fuerte, askha animuyuq, luka. Manan kankumanchu qharihina humildakuyta atinkumanchu. ["Les femmes auraient ces sept animu, les hommes au contraire en auraient peu. Les femmes sept, c'est pourquoi elles sont plus fortes, pleines d'animu, fofolles. Elles ne pourraient être comme les hommes, elles ne pourraient être humbles. »] (Anacleto Soto)

Si la présence de l'animu dans le corps garantit l'équilibre des humeurs, la possession de sept animu - comme c'est donc le cas pour les femmes - provoque un certain désordre et déséquilibre psychique qui se manifeste par leur caractère irascible et incontrôlable, ainsi que par une certaine fragilité émotionnelle, les rendant plus susceptibles de perdre l'animu (Figure 1).

En revanche les hommes, parce qu'ils ne possèdent que trois animu, sont plus forts et invulnérables au mensonge. Ils sont, de plus, moins susceptibles de perdre leur animu (Figure 2). Du moins, c'est ce que disent les femmes :

Qharikunaqa paykunaqa fuertepaschá. Chayrayku kimsa animullayuq ninku. Chayqa fuerte paykunaqa. Chayrayku mana ancha tanto facilta paykuna engañachikunmanchu. Manan qharikunata enganasunmanchu warmi riki. Chayqa 
anchayraykuyá chhayna. [« Les hommes, ils sont plus forts. C'est pour cette raison qu'on dit qu'ils n'ont que trois animu. C'est pour cette raison qu'ils sont forts et qu'on ne peut pas les tromper facilement. Nous, les femmes, nous ne pourrions pas tromper les hommes, n'est-ce pas! »] (Alejandrina Caylla)

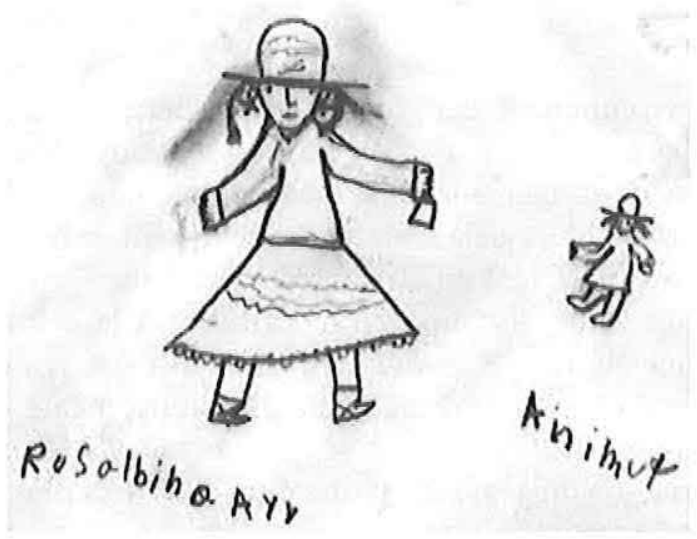

Fig. 1 - Représentation par une petite fille de la perte de l'animu chez une femme.

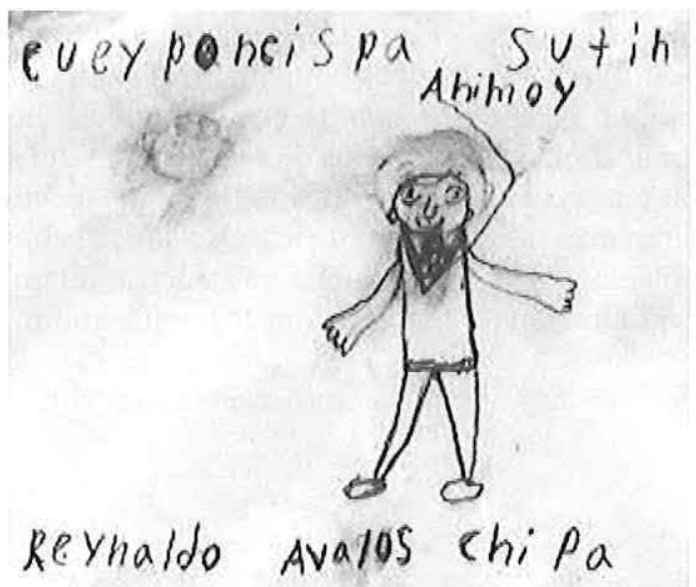

Fig. 2 - Représentation par un petit garçon de l'animu d'un homme.

Il nous paraît évident que les représentations et les discours relatifs à l'animu et à ses effets sur le corps et la personnalité des hommes et des femmes varient selon le genre des interviewés et sont destinés à légitimer les comportements ou le caractère de chacun des sexes. Ces représentations peuvent varier d'une région à 
l'autre. En effet, les représentations des substances animantes dans le nord du Pérou (Polia 1989) et en Bolivie (Fernández Juárez 1999), pour ne citer que ces deux régions, divergent de celles que nous venons d'étudier.

\section{CONCLUSION}

Nous avons vu comment, dans les Andes sud-péruviennes, la construction de l'identité sexuelle, étroitement associée aux conceptions relatives au corps et à la personne, débute avant même la naissance de l'individu, lors de rêves prémonitoires de la génitrice. Mais, déjà identifié sexuellement, le nouveau-né doit poursuivre sa construction en tant qu'être humain et qu'être sexué par le biais d'actes rituels de passage et de discours qui contribuent à le définir comme homme (qhari) ou femme (warmi). Ces différences doivent être renforcées et modelées par des pratiques rituelles qui modifient la nature même du corps et de la personne andine.

Cette construction du genre fait partie d'un processus plus général, celui de la construction de la personne humaine (runa) qui, également par le biais de rites et de discours, confère aux individus une nature à la fois sociale et sexuée. Tout nouveau-né est en effet considéré comme n'étant pas encore humanisé, donc comme étant saqra (" démon », « esprit maléfique »). Ce terme fait référence à la sphère du non socialisé, du sauvage et du nocturne, et s'oppose donc à ce qui est humain et domestique ${ }^{42}$. Devenir runa (« être humain »), homme ou femme, est le résultat instable d'un long processus de socialisation qui s'étend sur tout le cycle de vie et doit être systématiquement pour le moins réactualisé et surveillé. Par ailleurs, les différences essentielles entre les sexes sont à la base des rapports de dualité et de complémentarité (hiérarchique ou symétrique) qui caractérisent les rapports entre les hommes et les femmes dans le monde andin. *

* Manuscrit reçu en novembre 2008, accepté pour publication en juillet 2010.

\section{NOTES}

Je remercie Madame Catrysse, mon amie helléniste, pour sa relecture, ses corrections et observations toujours passionnantes.

1. En ce qui concerne l'évolution et les emplois du concept de " genre », ainsi que les différentes approches sur ce thème, voir Scott (1986), Conway et al. (1987).

2. Sur ce sujet, voir Delphy (2001) qui afiirme que le " genre précède le sexe » et que ce sont les rapports de pouvoir qui induisent la division sexuée de la société, non l'inverse.

3. Héritier (1996, p. 22) signale que "[...] les caractères observés dans le monde naturel sont décomposés, atomisés en unités conceptuelles, et recomposés dans des associations syntagmatiques qui varient selon les sociétés ". 
4. Pour un état des lieux assez complet à propos des études sur le genre et la parenté dans le monde andin, voir Arnold (1997, pp. 13-52; 1998).

5. Certains auteurs sont allés jusqu'à modifier leurs positions initiales. C'est le cas d'Isbell (1997) qui fait la critique de sa propre théorie qui postulait, en 1976, l'existence d'un « orden andino que es básicamente dual, complementario e igualitario » (Isbell 1976, p. 55). Sur ce thème, le lecteur pourra également se reporter aux textes de Spedding (1997), de Canessa (1997) et de Rösing (1997). Les notions de " dualité andine » et de " complémentarité andine » ont aussi été revisitées par Harris (2000). Nous ne retenons ici que les travaux concernant les communautés paysannes et rurales.

6. À notre connaissance, l'étude la plus complète sur la construction culturelle et sociale de l'enfant est celle de Lestage (1999), dans laquelle la logique de la fabrication de l'enfant est analysée sans se pencher toutefois spécifiquement sur son identité sexuelle. Pour une étude sur les représentations et les rituels de la grossesse chez les Macha qui établissent une analogie entre accouchement et transition mythico-historique, voir Platt (2001).

7. Surimana est situé près du fleuve Apurímac au nord-est de la province de Canas, à la frontière des provinces Chumbivilcas et Acomayo. Le terme employé par les commeros pour se référer à Surimana est celui de ayllu, terme qui désigne la communauté paysanne, mais aussi le lignage.

8. Le terme en espagnol pour désigner ces agents est promotores de salud. Il est intéressant de noter qu’à Surimana ce sont généralement les hommes qui remplissent le rôle d'accoucheurs. Il en va de même, dans la communauté Qulla d'Incawatana, au bord du lac Titicaca (Bolton et Bolton 1976, pp. 68,69 ). Cependant, aussi bien dans les sources anciennes que dans les études ethnographiques contemporaines portant sur le thème de l'accouchement dans les Andes, ce rôle est traditionnellement confié aux femmes. Pour les Xvi et xvir siècles, voir Polo de Ondegardo (1916 [1571], p. 35), Murúa (1987 [1613], livre 3, chap. LXII]), Guaman Poma (1980 [1615], pp. 192 [194]) et Cobo (1964 [1653], chap. XXXV). Pour la période actuelle, citons Seravia et Sueiro Cabredo (1985, p. 209), Tomaso et al. (1985, p. 207) et Bustos de Miranda (1977).

9. Nous traduisons par « infirmerie » ce qu'on désigne dans la région par posta médica, c'est-à-dire un établissement très modeste dans lequel sont dispensés les soins de base.

10. Ce réseau émet, sur les radios locales, des campagnes de prévention de santé en quechua.

11. La province de Canas est, en effet, une des plus pauvres au niveau national. De plus, les représentations concernant les politiques de santé officielles ne sont pas toujours positives. Le témoignage d'un accoucheur à ce sujet est explicite. Selon lui, la planificación familiar, c'est-à-dire les mesures de contrôle de la natalité, serait néfaste pour certaines femmes : "Chaypas wakinta allinta ruwan, efectuta ruwan. Wakintaqa astawan malta ruwan. Malta ruwan cuerpumta. Chayqa wakin senurakumataqa flacata ruwayapum. Pasaqta unquchipun astawan. Aha. Chay rayku wakin maman munankuchu chay planificación de familiarta. Wakinqa munakutaq. Wakintaqa provechanpunitaq chay planificación familiar " [ La planificación familiar a algumas les aprovecha, tiene efecto. En cambio a otras mucho daño les hace. Les hace mal a su cuerpo. La planificación familiar, a algumas señoras les hace bajar de peso. Totalmente les hace enfermar. Por eso algunas no quieren a esa planificación familiar) (Saturnino Mamani). En ce qui concerne les médicaments distribués par l'infirmerie, on peut entendre dire qu'ils "ne sont pas bons", qu'ils "ne guérissent pas»: "mana posta hampi allinchu. Manan thaninchimanchu » (Natividad Cuebas).

12. Notons que le terme runa est aussi employé pour faire référence aux populations autochtones des communautés paysannes par opposition aux Blancs et gringos. De même, dans la province de Churcapampa (département de Huancavelica), Magny (2007, p. 321) signale que le terme ruma, outre sa signification d'" homme " ou de "personne ", est utilisé par les métis "pour désigner les indigènes ayant choisi de ne pas se mélanger » avec les gringos et les métis. Cette acception d' "être humain » par opposition à ce qui n'est pas humain, mais aussi pour faire référence aux populations autochtones, est attestée depuis le Xvi ${ }^{e}$ siècle. Voir les désignations ethniques dans la Plática de Santo Tomas (1560), cité in Taylor (2001, p. 435). En aymara, l'équivalent de runa est jaqi « personne, individu, homme » (Rivière communication personnelle). Le verbe qui désigne le fait de « devenir homme » est jaqichasina, qui est le même terme que celui pour dire " casarse, convertirse así en persona » (Arnold et al. 1999, p. 102). 
13. Il est bon de noter qu'une nette distinction est faite entre les identités sexuelles humaines et animales. Les termes servant à désigner les mâles et les femelles étant respectivement urqu et china. Cette distinction s'inscrit dans un rapport plus général d'oppositions et d'équivalences entre le monde sauvage et le monde domestique.

14. Nous n'aborderons pas le rituel de la coupe de cheveux dans cet article. Signalons tout de même qu'avec ce rituel semble se mettre en place ce qu'on pourrait appeler la " domestication » de l'animu (en espagnol ánimo) et sa fixation dans le corps ; deux processus caractérisés par la maîtrise des sorties de celui-ci du corps lors d'expériences oniriques et par un contrôle de la parole. Pour une étude sur les représentations de l'animu, voir La Riva González (2005).

15. Sur les rencontres avec les gentils, à savoir les ancêtres de l'âge pré-solaire, dans les rêves, voir Robin (2008, p. 207).

16. Pour une étude sur les rêves " oraculaires » et leur rapport avec les rites d'initiation des spécialistes rituels, voir Fernández Juárez (1995a, p. 215; 1995b). L'initiation par les rêves n'est pas réservée qu'aux altumisayuq, au sommet de la hiérarchie des spécialistes rituels. Les accoucheurs et guérisseurs, hommes et femmes, peuvent aussi être initiés par des rêves au cours desquels des divinités révèlent des secrets thérapeutiques. C'est le cas du spécialiste rituel Anna Huallpa avec qui j'ai beaucoup travaillé. La révélation, par les rêves, des démarches thérapeutiques à suivre est attestée par Cobo $(1964$, p. 227) pour la période coloniale : « [... J diciendo que estando dormidos se les aparecio alguna persona, que, doliendose de su necesidad, les dijo que les daba facultad para curar de aquellas enfermedades que curaban; $y$ siempre que empezaban la cura, sacrificaban algo a aquella persona que afirmaba haberseles aparecido entre sueños y enseñandoles el modo de curar y los instrumentos con que lo habian de hacer » et mentionné par Fernández Juárez (1995a, p. 189).

17. Pour la transcription des racines verbales, je suis le travail d'Itier (s. d.). Elles sont suivies d'un tiret pour les différencier des noms.

18. Se reporter au dictionnaire de l'Academia Mayor de la lengua quechua (2005).

19. Depuis une vingtaine d'années, s'est développée une véritable anthropologie du rêve qui insiste sur les déterminants culturels des systèmes de rêves (voir les travaux de Tedlock 1987, 1991, 1994, ainsi que le numéro de 1996 de la revue Terrain. Rêver). Pour les rêves dans les sociétés amérindiennes, voir Perrin (1978). Pour le monde andin plus spécifiquement, voir Jiménez Borja (1961), Melis (1978), Zorrilla Eguren (1978), Mannheim (1987, 1991), Chávez (1996) et Andrade (2005).

20. Le motif de la reproduction des biens - plantes cultivées et animaux domestiques - à partir des fleurs offertes par une divinité, comme les esprits des montagnes ou la Pachamama, à un paysan pauvre, est récurrent dans la tradition orale (CBC 1990). Au XVII ${ }^{\mathrm{e}}$ siècle, les faucons et les vautours étaient des symboles oniriques qui annonçaient la naissance d'un enfant (Pérez Bocanegra 1631, pp. 127, 134). Ils apparaissent comme kamaq, " donneur de vie », des hommes et des femmes.

21. Sur les rites de fertilité des animaux, voir Aránguren (1975), Gow et Gow (1975).

22. Diosninchispa partinmanta sumaq sueñu, sumaq t'ikata rikunki [Les rêves provenant du coté de Dieu sont beaux, tu vois de belles fleurs].

23. Dans la zone de Marcapata, au sud de Cuzco, étudiée par Pablo Sendón, rêver de la bèche à pied, chakitaklla, annonce la naissance d'un garçon. Je tiens à remercier Pablo Sendón qui m'a permis d'avoir accès à son entretien avec Martin Quispe Rojas, paysan de Marcapata, au sujet de ses rêves: "[...] Chakitaklla [arado de pie] también es bueno, suerte. De repente vas a tener suerte y vas a tener hijo varón. Qhari waway. Chaymanta qhari wawayoq kanaykipaq /Hijo varón. Para que puedas tener hijo varón.] Instrumento también. Ishkay t'aqamanta qhari wawayoq kanaykipaq /Para que puedas tener hijo varón de dos grupos] De banda, bajo, de trompeta, de un algo. Entonces, eso [... ] para que tengas hijos. Asi habia sido » (Pablo Sendón communication personnelle). Une miniature comme une chakitaklla peut être enterrée avec le placenta d'un garçon, comme nous le verrons plus loin.

24. Il serait intéressant de se pencher sur le rapport d'équivalence entre fille et filleul, mais pour le moment nous n'avons que très peu d'éléments.

25. En espagnol dans l'entretien. 
26. En quechua, $q^{\prime} u r u t a$ signifie testicule et, en aymara, ce même terme désigne à la fois la graine ou le pépin/noyau d'un fruit et les testicules (Gilles Rivière communication personnelle).

27. Les graines de maïs, de qañiwa (Chenopodium pallidicaule) et les huairuru (wayruru en quechua, plante de la famille des légumineuses, Ormosia coccinea) font partie des offrandes aux morts, mais aussi des offrandes faites à la Pachamama et aux Apu dans ce qu'on appelle les despachos. Pour les offrandes aux morts, voir Santos (1988). Dans la culture Paracas Necropolis (200 av.-200 apr. J.-C.), des graines de haricots noirs ont été trouvées dans les fardeaux funéraires de spécialistes rituels comme cela a été montré lors de l'Exposition «Paracas. Trésors inédits du Pérou ancien », musée du quai Branly, avril-juillet 2008).

28. L'équivalence entre sperme et graine est explicite dans une des expressions utilisées pour se référer aux hommes stériles chak'i sara "maïs sec ». Au XvI" siècle, nous trouvons cette équivalence entre aliment concentré (graine) et sperme dans la chapitre II des Rites et traditions de Huarochiri où l'on raconte le mythe de Cuniraya Huiracocha et Cahuillaca, dans lequel Cahuillaca engendre un enfant de Huiracocha après avoir mangé un fruit de lucuma à l'intérieur duquel Huiracocha avait déposé son sperme (Taylor 1987, p. 55). Pour une équivalence entre graine et testicule en aymara, voir supra note 26.

29. Par exemple, chocar («frapper » en espagnol) est un des termes repris et fait référence au sperme qui pénètre l'ovaire, évoquant les discours médicaux officiels sur la reproduction.

30. Les fetus issus d'une fausse couche et les morts-nés reçoivent aussi ce traitement rituel car, autrement, ils se transformeraient en duende ( mauvais esprit »), réincorporant le ventre de la mère et provoquant la maladie ou la mort. Duende ninku riki mana unuchasqataqa. Chayqa senuraq wiksanmansiya kasqanta chay mal espiritu kutiyapullantaq chay wawachaq mal espiritun. Chayqa wiksapi creakapullantaq. Chay señurata, mamitanta malta ruwapun. Chayqa unquyullantaq. Chaymi mana allinchu [" Les enfants à qui l'on n'a pas donné l'ondoiement, on les appelle duende. On dit que ce mauvais esprit retourne dans le ventre de la mère, c'est-à-dire le mauvais esprit du bébé. Ainsi il se reconstitue dans l'estomac; cela fait mal à la mère. Elle pourrait tomber enceinte ou tomber malade. $\mathrm{Ce}$ n'est pas bon "] (Saturnino Mamani).

31. Platt (2001, p. 649) signale aussi un comportement différencié selon le genre en fonction des mouvements perçus par la mère dans son ventre et de la constitution du fæetus masculin et féminin.

32. Platt (2001, p. 649) rapporte également que les garçons sont comme des petits poissons ou challwa.

33. Le sexe masculin est connu sous différents noms : pichiku, ch'unchulin, hatun pichiku, huchuy pichiku, ullu, pisqu.

34. En tant que double de la mère, le placenta est responsable des taches au visage des femmes: mancha uyaykipi lluqsin chayqa, yawarkunata maqchi maqchirispa sumaqta urqupuyku [« il y a des taches qui apparaissent sur ton visage, lorsque nous lavons bien le sang du placenta elles disparaissent »]. Double du nouveau-né, mes interlocuteurs disent que, si l'on ne lave pas bien le placenta, le nouveau-né devient vieux et la peau toute noire. Palmira La Riva : Mana kanasqa imataq pasan? [" et quand on ne le brûle pas (le placenta), qu'est-ce qui arrive? »]. Natividad Cuebas : Imayá pasakumman, punkillikunkus ninku, chaymanta nan, iman, wawa yana machuchamansi tukupun mana maqchisqa mana paris maqchhisqa ninku, yana chilluchamansi wasa tukupun ninku mana parista maqchhiqtiyki, limphuyta maqchhinki parista anchaysi munay zorrocha wawaqa, mana maqchhisqaqa yana machusyá [" Je ne sais pas ce qui pourrait arriver. On dit qu'on se gonfle, d'autres que le bébé devient comme un vieux noir parce qu'on n'a pas lavé le placenta. On dit que le bébé devient noir, très noir. On dit que, quand on lave bien le placenta, le bébé est blond. Si l'on ne le lave pas, il est comme un vieux noir $»]$.

35. Le « mauvais air » renvoie à une catégorie de maladies liée à toutes sortes d'airs et de vents pathogènes pouvant s'introduire dans le corps. Pour plus d'informations sur ce sujet, voir La Riva (2005, p. 65).

36. L'enfant à qui on ne fait pas le umuchay (l'ondoiement), acte rituel qui le transforme en personne (ruma), lui donnant un nom, peut aussi vouloir réintégrer le ventre de la mère et la tuer. Il est 
appelé, dans ce cas, duende « mauvais esprit ». Un enfant mort-né doit aussi bénéficier de cet acte rituel. Les personnes avec lesquelles j'ai travaillé disent qu'autrefois on le brûlait au sommet des montagnes : Nawpaq ichaqa kanaqku duende nispa, urqu puntakunaman, antes kanaqku. Kunanqa mana hinachu. Maman [" Même si tu accouches d'un petit mort-né, il faut toujours faire l'ondoiement. Avant on avait la coutume de le brûler, on l'emmenait au sommet des montagnes et on le brûlait. Maintenant on ne fait plus cela »] (Saturnino Mamani). Davidson (1983, p. 72) indique que, dans le nord du Pérou, le placenta peut produire "l'air du mort", qui compte parmi les maladies répertoriées dans la médecine andine.

37. Pour une description minutieuse des étapes de la fabrication rituelle du mort dans deux communautés sud-andines, voir Robin (2008). Cet auteur rapporte un traitement différencié de la coiffure du mort selon qu'il s'agit d'un homme ou d'une femme (ibid., p. 70).

38. L'animu joue un rôle fondamental dans les représentations du corps et de la personne dans le monde andin. Cette substance vitale maintient le corps en vie en garantissant la circulation de ses humeurs. Pour une étude sur les représentations de l'amimu, voir La Riva González (2005).

39. Dans la communauté d'Andamarca (département d'Ayacucho), Ossio (1992, p. 282) mentionne une différenciation dans les pratiques funéraires selon que le mort est un homme ou une femme : se repican las campanas tres veces y cuando son mujeres, siete veces, correspondiendo con el número de almas que se supone tiene cada sexo ["lorsqu'il s'agit d'hommes, les cloches sonnent trois fois et, lorsqu'il s'agit de femmes, elles sonnent sept fois, en fonction du nombre d'âmes que chacun des sexes est supposé avoir »].

40. Se reporter au dictionnaire de l'Academia mayor de la lengua quechua (2005).

41. Les femmes interrogées disent aussi que les hommes sont comme le vent wayrahina.

42. Dans une perspective mythico-historique, Platt (2001) analyse le parallélisme entre la formation de la personne et l'avènement d'une société chrétienne.

\section{RÉFÉRENCES CITÉES}

Academia mayor de la lengua quechua

2005 Diccionario Quechua-Español-Quechua. Simi taqe, Gobierno Regional, Cuzco.

ANDRADE Luis

2005 Aguas turbias, aguas cristalinas: el mundo de los sueños en los andes surcentrales, Fondo Ed. de la Pontificia Universidad Católica del Perú, Lima.

Aránguren Paz A.

1975 "Las creencias y ritos mágicos religiosos de los pastores puneños », Allpanchis Phuturinqa, 8, pp. 103-132.

ARNOLD Denise

1997 «Introducción », in Denise Arnold (éd), Más allá del silencio. Las fronteras de género en los Andes, CIASE/ILCA, La Paz, pp. 13-52.

ARnold Denise (éd.)

1998 Gente de carne y hueso. Las tramas de parentesco en los Andes, CIASE/ILCA, La Paz.

Arnold Denise, Juan de Dios Yapita et Margarita Tito

1999 Vocabulario aymara del parto y de la vida reproductiva de la mujer, ILCA/FHI, La Paz. 
Bolton Ralph et Catherine Bolton

1976 «Concepción, embarazo y alumbramiento en una aldea Qolla », Antropología Andina, 1 (2), pp. 58-73.

BouRdieu Pierre

1998 La domination masculine, Seuil, Paris.

Bustos de Miranda Julia

1977 El parto tradicional [manuscrit].

CANEssa Andrew

1997 "Género, lenguaje y variación en Pocobaya, Bolivia », in Denise Arnold (éd.), Más allá del silencio. Las fronteras de género en los Andes, CIASE/ILCA, La Paz, pp. 233-250.

CBC (Centro Bartolomé de las Casas)

1990 Cuentos campesinos. Khuyapayakuq Apu y otros cuentos (quechua), Centro de estudios regionales andinos, Cuzco.

CERrón-PALOMino Rodolfo

1994 Quechua sureño, diccionario unificado, Biblioteca Nacional del Perú, coll. « Biblioteca Básica Peruana », Lima.

CHÁvez Fabiola

1996 «Iniciación y sueño entre las "parteras" de la sierra de Piura (Ayabaca) », Anthropológica, 14, pp. 183-207.

Christinat Jean-Louis

1989 Des parrains pour la vie. Parenté rituelle dans une communauté des Andes péruviennes, Institut d'Ethnologie, Neuchâtel/Maison des sciences de l'homme, Paris.

Coвo Bernabé

1964 Historia del Nuevo Mundo, 2 volumes, Biblioteca de Autores Españoles, Madrid [1653].

Conway Jill K., Susan C. Bourque et Joan W. ScotT (éds)

1987 Learning about women : gender, politics and power, The University of Michigan Press, Ann Arbor.

DAVIDSON Judith R.

1983 «La sombra de la vida : la placenta en el mundo andino », Bulletin de l'Institut français d'études andines, XII (3-4), pp. 69-81.

Delphy Cristine

2001 L'ennemi principal, tome 2: Penser le genre, Syllepsi, Paris.

\section{Dopds Eric Robertson}

1966 "Dream pattern and culture pattern », in Eric Robertson Dodds, The Greeks and the irrational, University of California Press, Berkeley/Los Angeles, pp. 102-134. 


\section{FERNÁNDEZ JuÁREZ Gerardo}

1999 Médicos y yatiris. Salud e interculturalidad en el Altiplano Aymara, Centro de Investigación y Promoción del Campesinado, coll. « Cuadernos de Investigación » 51, La Paz.

1995a El banquete aymara. Mesas e yatiris, Hisbol, La Paz.

1995b «Imágenes, intelecto, palabras : el ojo del sueño en la formación de un "maestro" ceremonial aymara ", Revista Andina, 13 (26), pp. 389-420.

\section{Foucault Michel}

1984 Histoire de la sexualité, vol. 2 : L'usage des plaisirs; vol. 3 : Le souci de soi, Gallimard, Paris.

\section{Godelier Maurice et Michel Panoff (éds)}

1998 La production du corps. Approches anthropologiques et historiques, Éditions des archives contemporaines, Amsterdam.

\section{Gow David et Rosalinda Gow}

1975 «La alpaca en el mito y el ritual », Allpanchis Phuturinqa, 8, pp. 141-162.

\section{Guaman Poma de Ayala Felipe}

1980 Nueva Corónica y Buen Gobierno, Biblioteca Ayacucho, Caracas [1615].

HARrIS Olivia

1980 "The power of signs ", in Carol MacCormack et Marilyn Strathern (éds), Nature, culture and gender, Cambridge University Press, Cambridge, pp. 70-95.

2000 "Complementarity and conflict : an Andean view of women and men ", in Olivia Harris, To make the earth bear fruit : ethnographic essays on fertility, work and gender in Highland Bolivia, Institute of Latin American Studies, Londres, pp. 164-179.

\section{HéRITIER Françoise}

1996 Masculin/Féminin. La pensée de la différence, Odile Jacob, Paris.

ISBELL Billie Jean

1976 "La otra mitad esencial : un estudio de complementariedad sexual andina », Revista de Estudios Andinos, 5 (1), pp. 37-56.

1997 «De inmaduro a duro : lo simbólico femenino y los esquemas andinos de género », in Denise Arnold (éd.), Más allá del silencio. Las fronteras de género en los Andes, CIASE/ILCA, La Paz, pp. 253-301.

\section{ITIER César}

s. d. Dictionnaire quechua-français [à parâtre].

\section{JIMÉNEZ BORJA Arturo}

1961 «La noche y el sueño en el antiguo Perú », Revista del Museo Nacional, 30, pp. 84-94.

La Riva Gonzalez Palmira

2000 "Le walthana hampi ou la reconstruction du corps. Conception de la grossesse dans les Andes du Sud du Pérou ", Journal de la Société des Américanistes, 86, pp. 169-184. 
2005 "Las representaciones del animu en los Andes », Revista Andina, 41, pp. 63-88.

Lestage Françoise

1999 Naissance et petite enfance dans les Andes péruviennes. Pratiques, rites, représentations, L'Harmattan, Paris.

MaGny Caroline

2007 Boire à Churcampa (Pérou) : de la production des boissons au sens de l'ivresse, thèse, université Paris X-Nanterre, Nanterre.

MEAD Margaret

1963 Maurs et sexualité en Océanie, Plon, Paris [1928].

MANNHEIM Bruce

1987 "A semiotic structure of Andean dreams », in Barbara Tedlock (éd.), Dreaming. Anthropological and psychological interpretations, Cambridge University Press, Cambridge, pp. 132-156.

1991 "After dreaming: image and interpretation in Southern Peruvian Quechua », Etnofoor, IV (2), pp. 43-79.

Melis Paola

1978 "Sueños, conciencia y niveles cósmicos en el "curanderismo" del Perú septentrional ", in Michel Perrin (éd.), Antropología y experiencias del sueño, Abya-Yala/MLAL, Quito, pp. 159-178.

Murúa Martín de

1987 Historia general del Perí, edición de Manuel Ballesteros/Historia 16, Madrid [1613].

Ossio Juan

1992 Parentesco, reciprocidad y jerarquía en los Andes: una aproximación a la organización social de la comunidad de Andamarca, Pontificia Universidad Católica/Fondo Editorial, Lima.

PÉrez Bocanegra Juan

1631 Ritual formulario e institución de curas, para administrar a los naturales de este Reyno los Santos Sacramentos del baptismo, confirmación, eucaristía, y viatico, penitencia, extremaunción y matrimonio, con aduertencias muy necesarias, Gerónimo de Contreras, Lima.

PERrin Michel

1978 Antropología y experiencias del sueño, Abya-Yala/MLAL, Quito.

Platt Tristan

2001 «El feto agresivo. Parto, formacion de la persona y mito-historia en los Polia Mario Andes ", Anuario de Estudios Americanos, LVIII (2), pp. 663-678.

1989 " "Contagio" y "pérdida de la sombra" en la teoría y práctica del curanderismo andino del Perú septentrional : provincias de Ayabaca y Huancabamba ", Anthropologica, 7, Lima, pp. 195-231. 


\section{Polo de ONDEgardo Juan}

1916 Informaciones acerca del la religión y gobierno de los incas. $1^{a}$ parte, Imprenta y librería San Martí, coll. «Colección de libros y documentos referentes a la historia del Perú "), ${ }^{\text {re }}$ série, t. III, Lima [1571].

RoBIN Valérie

2008 Miroirs de l'autre vie. Pratiques rituelles et discours sur les morts dans les Andes de Cuzco (Pérou), Société d'ethnologie, coll. "Recherches Américaines $\gg 7$, Nanterre.

RösING Ina

1997 "Los diez géneros de Amarete, Bolivia », in Denise Arnold (éd.), Más allá del silencio. Las fronteras de género en los Andes, CIASE/ILCA, La Paz, pp. 77-93.

\section{SANTOS Felipe}

1988 «La fiesta de difuntos en un contexto suburbano. La muerte ritual de los ajayu », Fe y Pueblo, 19, Centro de Teología Popular, La Paz, pp. 34-43.

\section{ScotT Joan W.}

1986 «Gender : a usefull category of historical analysis », American Historical Review, 91, pp. 1053-1075.

Seravia Luis Miguel et Rosa Sueiro Cabredo (éds)

1985 Experiencias de desarrollo popular en el campo de la medicina tradicional y moderna, DESCO/Centro de Estudios y Promoción del Desarrollo, coll. «Serie experiencias de desarrollo popular », Lima.

\section{SPEDDING Alison}

1997 «Esa mujer no necesita hombre: en contra de la "dualidad andina". Imágenes de género en los yungas de la Paz », in Denise Arnold (éd.), Más allá del silencio. Las fronteras de género en los Andes, CIASE/ILCA, La Paz, pp. 325-343.

\section{TAYLOR Gérald}

1987 Ritos y tradiciones de Huarochirí : manuscrito quechua de comienzos del siglo xVII, estudio biográfico sobre Francisco de Ávila de Antonio Acosta, Instituto de Estudios Peruanos/Institut français d'études andines, Lima.

2001 "La Plática de Fray Domingo de Santo Tomás (1560) », Bulletin de l'Institut francais d'études andines, 30 (3), pp. 427-453.

TEDLOCK Barbara

1987 Dreaming : anthropological and psychological interpretations, Cambridge University Press, Cambridge.

1991 "The new anthropology of dreaming », Dreaming, 1 (2), pp. 161-178.

1994 "Rêves et visions chez les Amérindiens : produire un ours 》, Anthropologie et Sociétés, 18 (2), pp. 13-27.

Terrain

1996 Rêver, 26. 
Tomaso Diana, Andrea CAPrara et Eduardo ChiESA

1985 «Trabajo con parteras tradicionales en la provincia de Grau-Apurímac. Equipo socio-sanitario de la Prelatura de Chuquibambilla, Provincia de Grau, dept. Apurímac ", in Luis Miguel Seravia et Rosa Sueiro Cabredo (éds), Experiencias de desarrollo popular en el campo de la medicina tradicional y moderna, DESCO/Centro de Estudios y Promoción del Desarrollo, coll. «Serie experiencias de desarrollo popular », Lima, pp. 201-225.

ZORRILLA EgUREN Javier

1978 "Sueño, mito y realidad en una comunidad ayacuchana », Debates en antropologia, 2, pp. 119-125. 